\title{
ANASTOMOSIS URETEROINTESTINALES. NUESTRA EXPERIENCIA. CUANTO MÁS SIMPLE...MEJOR
}

\author{
J.L. MOYANO CALVO, J. CASTIÑNIRAS FERNÁNDEZ \\ Cátedra y Servicio de Urología del Hospital Universitario Virgen Macarena. \\ Fundación Joaquín Albarrán. Sevilla. \\ Actas Urol Esp. 27 (9): 713-717, 2003
}

\section{RESUMEN}

ANASTOMOSIS URETEROINTESTINALES. NUESTRA EXPERIENCIA. CUANTO MÁS SIMPLE...MEJOR INTRODUCCIÓN: Están descritas numerosas técnicas para el reimplante ureterointestinal. Presentamos nuestra evolución con tres de ellas con la intención de disminuir la incidencia de estenosis. MATERIAL Y MÉTODO: 77 pacientes a los que hemos realizado cistectomía radical y derivación urinaria con intestino delgado en 20 pacientes (neovejiga tipo Paduana) o con sigma destubulizado en 57 (Mainz II). Inicialmente realizábamos anastomosis según técnica de Le Duc, posteriormente puño de camisa (Ricard) y actualmente anastomosis tipo bivalva.

RESULTADOS: Hemos realizado anastomosis tipo Le Duc en el $38 \%$ y puño de camisa en el $31 \%$, bivalva en el $27 \%$ de las vías urinarias. Seguimiento medio 37,7 meses, 19,5 meses, y 10 meses respectivamente.

Estenosis: 16,7\% con técnica Le Duc; 20,5\% con técnica Ricard; 9,1\% con técnica Bivalva. Anulación renales: 10,4\% con técnica Le Duc; 10,3\% con técnica Ricard; 0\% con técnica Bivalva (p .000).

CONCLUSIONES: En nuestra experiencia la anastomosis menos estenosante es la tipo bivalva.

A mayor sencillez de técnica mejores resultados.

La tasa de estenosis es siempre mayor cuando la vía está dilatada previa a la derivación, independientemente de la técnica utilizada.

PALABRAS CLAVE: Estenosis. Anastomosis ureterointestinal. Neovejiga.

\section{ABSTRACT}

URETEROENTERIC ANASTOMOSES. OUR EXPERIENCE. THE SIMPLIEST, THE BEST

INTRODUCTION: There are many techiques describe for ureteroenteric anastomoses. We present our experience with three of them inorder to reduce the stenosis rate.

METHOD AND MATERIAL: We have done radical cystectomy in 77 patients. Urinary diversion with small bowel in 20 patients (Paduana ileal neobladder) and dstubulized ureterosigmoidostomy (MainzII) in 55.

Initialy, we used Le Duc technique, afterward Ricard technique and actually bivalva technique (direct).

RESULTS: We have performed Le Duc in 38\%, Ricard in 31\% and "bivalva" in 27\%. Median follow-up are 37,7 months, 19,9 months and 10 months respectivily.

Stenosis: $16,7 \%$ with Le Duc; $20,5 \%$ with Ricard and 9,1\% with bivalva technique. Renal anulation: $10,4 \%$ with Le Duc; $10,3 \%$ with Ricard and $0 \%$ with bivalva (p .000).

CONCLUSIONS: In our experience, the less stenosant anastomose is BivalvaIn one.

The simpliest technique, the best results. The rate of stenosis is higher when the upper urinary tract is dilatated pre-surgery, independently of the anastomosis technique.

KEY WORDS: Stenosis. Ureteroenteric anasatomose. Noebladder. 
$\mathrm{S}_{\mathrm{m}}^{\mathrm{i}}$ inicialmente el reflujo era la complicación más temida de las anastomosis ureterointestinales, describiéndose numerosas técnicas anastomóticas antirreflujo, desde el uso de segmentos intestinales destubulizados es la estenosis de la unión ureterorintestinal la complicación más seria de la misma.

Presentamos la evolución de las técnicas de anastomosis ureterointestinal en nuestro Servicio, buscando la menos estenosante.

Inicialmente realizábamos anastomosis según técnica de Le Duc (Fig. 1), posteriormente puño de camisa o Ricard (Fig. 2) y actualmente anastomosis directa tipo bivalva (Fig. 3).

\section{MATERIAL Y MÉTODO}

Son valorables 77 pacientes a los que hemos practicado cistectomía radical y derivación urinaria. Hemos realizado:

- Neovejiga con íleon destubulizado tipo Paduana en 20 pacientes.

- Ureterosigmoidostomía destubulizada tipo Mainz II en 57 pacientes.

Los enfermos han sido valorados mediante urografía intravenosa, ecografía y renograma isotópico con test de Furosemida.

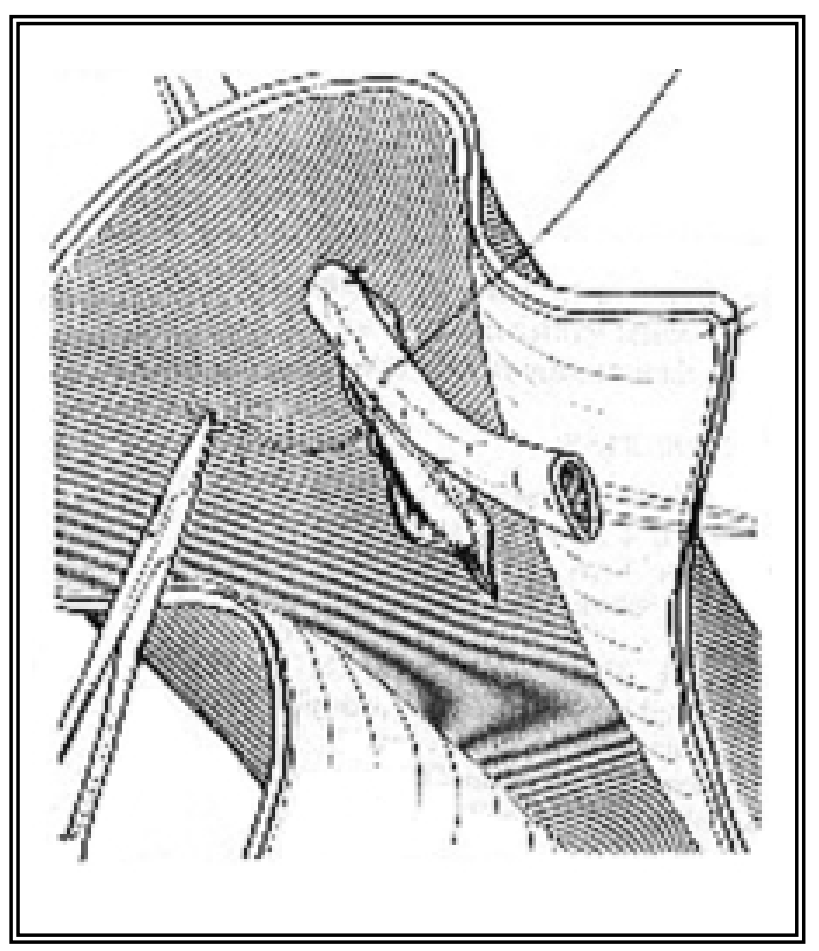

FIGURA 1. Anastomosis según técnica de Le Duc.

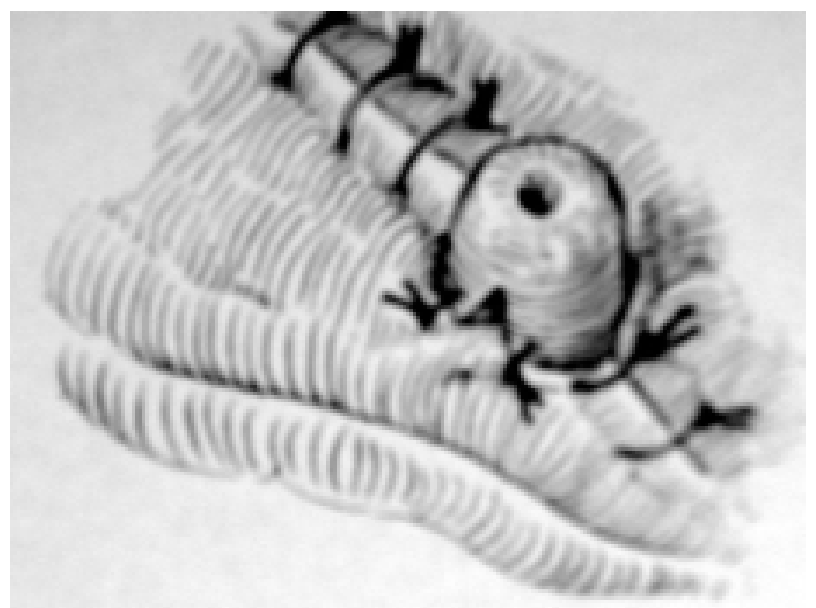

FIGURA 2. Anastomosis según técnica de Ricard.

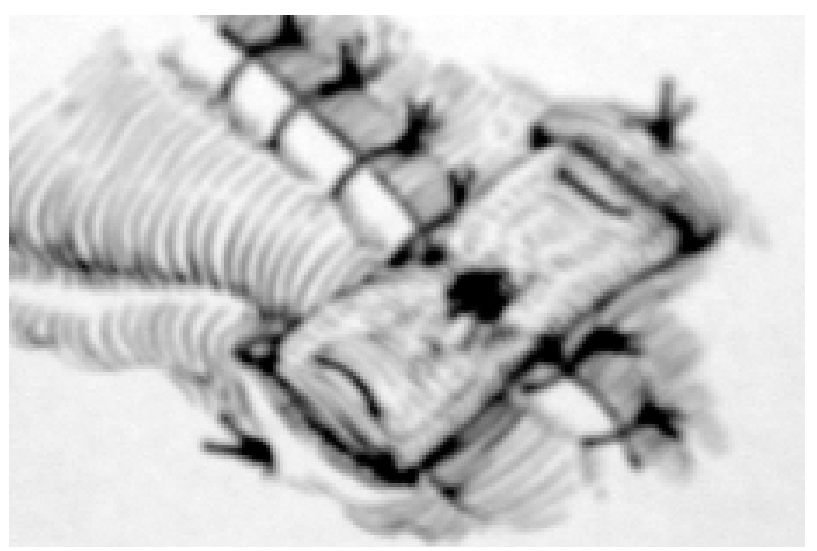

FIGURA 3. Anastomosis según técnica Bivalva (Lichtenauer o Puigvert).

En todos los casos se disecó el uréter lo menos posible para evitar isquemias. Las anastomosis se realizaron con sutura $4 / 0$ de material reabsorbible (ácido poliglicólico).

En las derivaciones tipo Mainz II el reimplante se realizó transcolónico completamente extraperitoneal.

En el análisis estadístico se ha utilizado el estadístico Chi-cuadrado.

Seguimos los criterios de O'Reilly ${ }^{1}$ para diferenciar entre vía dilatada (responde a diurético) y vía estenótica (no responde a diurético).

\section{RESULTADOS}

Los tipos de anastomosis realizados son:

- Tipo Le Duc........................................38\%

- Tipo Ricard o "Puño de camisa" .............31\%

- Tipo "Bivalva" ......................................27\% 
El seguimiento medio es 37,7 meses, 19,5 meses y 10 meses respectivamente.

El resultado en conjunto se muestra en la Tabla I. Como podemos apreciar el 16,7\% de las vías a las que se les ha realizado reimplante ureteral tipo Le Duc presentan estenosis frente al 20,5\% de las anastomosis tipo "Puño de camisa" y el 9,1\% de las tipo "Bivalva". Ninguna de las vías reimplantadas con esta última técnica presentan anulación en el renograma isotópico. La diferencia es estadísticamente significativa (p .000).

Los resultados para lado derecho e izquierdo en las Tablas II y III, siendo la significación estadística p .000 para el lado derecho y p .000 para el izquierdo.

\section{TABLA I}

RESULTADOS EN CONJUNTO DE AMBAS VÍAS URINARIAS

\begin{tabular}{||l|c|c|c|c||}
\hline & Normal & Dilatada & Estenosis & Anulada \\
\hline Le Duc & $56,3 \%$ & $16,7 \%$ & $16,7 \%$ & $10,4 \%$ \\
\hline Ricard & $51,3 \%$ & $17,9 \%$ & $20,5 \%$ & $10,3 \%$ \\
\hline Bivalva & $57,6 \%$ & $33,3 \%$ & $9,1 \%$ & - \\
\hline
\end{tabular}

TABLA II

RESULTADOS VÍA URINARIA DERECHA

\begin{tabular}{|l|c|c|c|c|}
\hline & Normal & Dilatada & Estenosis & Anulada \\
\hline Le Duc & $52 \%$ & $12 \%$ & $16 \%$ & $16 \%$ \\
\hline Ricard & $53,3 \%$ & $20 \%$ & $26,7 \%$ & - \\
\hline Bivalva & $57,1 \%$ & $33,3 \%$ & $9,5 \%$ & - \\
\hline
\end{tabular}

TABLA III

RESULTADOS VÍA URINARIA IZQUIERDA

\begin{tabular}{|l|c|c|c|c||}
\hline & Normal & Dilatada & Estenosis & Anulada \\
\hline Le Duc & $60,9 \%$ & $21,7 \%$ & $17,4 \%$ & - \\
\hline Ricard & $50 \%$ & $16,7 \%$ & $16,7 \%$ & $16,7 \%$ \\
\hline Bivalva & $58,3 \%$ & $33,3 \%$ & $8,3 \%$ & - \\
\hline
\end{tabular}

\section{DISCUSION}

La complicación post-quirúrgica más importante y con mayores efectos secundarios sobre la unidad renal es la estenosis de la anastomosis ureterointestinal. Esta se presenta entre el 2 y el
$30 \%$ de las series, dependiendo de la técnica realizada y del seguimiento de la misma². La mayoría de las estenosis se producen en los primeros meses tras la derivación, pero se han descrito estenosis tardías a los 14 años $^{3}$.

La estenosis ureterointestinal tiene una etiología multifactorial, influyendo:

- Aspectos técnicos en la ejecución de la anastomosis (torsión del uréter, aposición mucosa-mucosa, calibre de sutura utilizada...).

- Isquemia del uréter.

- Radioterapia.

- Infecciones.

- Fuga de orina por fístula a nivel anastomótico.

Se han descrito numerosas técnicas de anastomosis ureterointestinal, pero básicamente se pueden dividir en dos grupos dependiendo en que contemplen o no la posibilidad de prevenir el reflujo urinario.

Los reservorios que actualmente configuramos son de baja presión debido a la destubulización del intestino. Por ello, el reflujo, complicación muy temida con las derivaciones tubulizadas donde se alcanzan presiones dentro del reservorio superiores a $60 \mathrm{~cm}$ de $\mathrm{H} 2 \mathrm{O}$, ha dejado de ser un problema ya que raramente se alcanzan presiones superiores a $40 \mathrm{~cm}$ de $\mathrm{H}_{2} \mathrm{O}^{4-6}$ consideradas lesivas para el aparato urinario superior ${ }^{7}$. Por ello hoy día se plantea si es necesario realizar técnicas antirreflujo en este tipo de derivaciones ${ }^{8-11}$, dado que la adopción de las mismas:

Aumenta el riesgo de estenosis. Aspecto reconocido por la mayoría de los autores. Así:

Roth en 120 pacientes realiza técnica de Le Duc en 142 uréteres, con un 20,4\% de estenosis frente al $3,6 \%$ en 83 uréteres implantados con técnica de anastomosis directa tipo Nesbit ${ }^{10}$. Pantuck en 58 pacientes, realiza técnicas antirreflujo en 50 uréteres, con un $13 \%$ de estenosis frente al $1,7 \%$ de aquellos en que realiza técnica directa tipo Nesbit ${ }^{8}$. Helal, en 190 pacientes observa $13 \%$ de estenosis con técnica tunelizada tipo Goodwin frente a 4,9\% usando anastomosis directa $^{11}$. Kristjannson en 56 pacientes no aprecia diferencias en la tasa de estenosis dependiente de la técnica anastomótica ${ }^{3}$. De Carli en 102 pacientes no aprecia diferencias en cuanto a estenosis $(7 \%$ frente a $6,7 \%)$ con reimplante tipo puño de camisa o anastomosis directa ${ }^{9}$. 
No previene completamente el reflujo. De Carli en 102 pacientes no aprecia diferencias en cuanto a reflujo (20\% frente a $23 \%)$ con reimplante tipo puño de camisa o anastomosis directa ${ }^{9}$. Le Duc comunica cifras de reflujo de $19 \%{ }^{12}$. Kristjansson aprecia daño renal secundario a reflujo en el 33\% de los conductos colónicos o ileales y en el $63 \%$ de los reservorios cecales a pesar de realizar técnica antirreflujo ${ }^{13}$.

Bajo tasa de deterioro renal. La tasa de deterioro renal comunicada por los distintos autores, es muy baja. Así Hautmman, que realiza anastomosis tipo Le Duc tiene $0,8 \%$ de insuficiencia renal crónica de un total de 363 pacientes ${ }^{14}$. Thoney que realiza la anastomosis tipo Nesbit sólo observa un $1 \%$ de deterioro renal causado por estenosis de la unión ureterointestinal, pero no por el reflujo ${ }^{15}$. Kristjansson no encuentra un efecto adverso del reflujo sobre la función renal tras 123 meses de seguimiento medio ${ }^{3}$. Golbano y Fisch tampoco refieren deterioro de la función renal en derivaciones tipo Mainz $\mathrm{II}^{4,16}$.

El mecanismo antirreflujo no tiene por qué depender de la técnica anastomótica. Tanto Thoney ${ }^{15}$ que utiliza un asa isoperistáltica como Skinner ${ }^{17}$ que utiliza un segmento de asa intususceptada, realizan anastomosis directas con una bajísima tasa de estenosis ( $1 \%$ y $3 \%$ ) y con práctica ausencia de reflujo urinario. Studer ${ }^{18}$ compara ambas técnicas y las dos son igual de eficaces en condiciones fisiológicas

En nuestra experiencia, la adopción de una técnica anastomótica directa ha reducido la tasa de estenosis en más de la mitad, situándola en un $9 \%$.

La técnica de Le Duc presenta una tasa de estenosis que varía entre un $1,5 \%^{12}$ y un $29 \%{ }^{19}$. La variación tan amplia de resultados es explicable por varias teorias: experiencia de cirujanos, reacción inflamatoria de la adventicia ureteral, cambios inflamatorios en la pared ureteral. Para mejorar la tasa de estenosis han sido descritas al menos tres modificaciones técnicas ${ }^{20-22}$.

Nuestros resultados, $16,7 \%$ de estenosis, se sitúan dentro de los comunicados por la literatura.

Con la técnica de Ricard o Turner-Warwick o puño de camisa la tasa de estenosis oscila entre $0 \%$ (Stone) ${ }^{23}$ y $7 \%{ }^{10}$. Nuestra tasa de estenosis es excesivamente alta $(20,5 \%)$. Creemos que es atribuible al alto número de vías dilatadas previas a la cirugía que se reimplantaron con este método. Como podemos apreciar en las Tablas IV y V el número de vías dilatadas es sensiblemente mayor en los que se efectuó técnica de Ricard, así como que la tasa de estenosis es siempre mayor, independientemente de la técnica, cuando la vía está dilatada. Sagalowsky ${ }^{24}$ disminuye su tasa de estenosis confeccionando el puño de camisa antes de atravesar la pared intestinal y ampliando la longitud de espatulización. Marino ${ }^{25}$, disminuye de $6,8 \%$ a $2,9 \%$ las estenosis al resecar un botón de intestino en vez de realizar una enterotomía simple.

\section{TABLA IV}

RELACIÓN DEL ESTADO DE LA VÍA PREVIA A CIRUGÍA CON LA TÉCNICA ANASTOMÓTICA

\begin{tabular}{|l|c|c|c|}
\hline \multirow{2}{*}{} & \multicolumn{2}{|c|}{ Vía Previa a Cistectomía } & \multirow{2}{*}{ p } \\
\cline { 2 - 3 } & Normal & Dilatada & \\
\hline Le Duc & $93,5 \%$ & $6,5 \%$ & p.009 \\
\hline Ricard & $57 \%$ & $43 \%$ & p.577 \\
\hline Bivalva & $78,4 \%$ & $21,6 \%$ & p.024 \\
\hline
\end{tabular}

TABLA V

TASA DE ESTENOSIS SEGÚN TÉCNICA ANASTOMÓTICA Y ESTADO DE LA VÍA PREVIO A CIRUGÍA

\begin{tabular}{||l|c|c|c|c|}
\hline & Le Duc & Ricard & Bivalva & p \\
\cline { 1 - 4 } Normal & $14 \%$ & $13,6 \%$ & $3,4 \%$ & \multirow{2}{*}{ p.000 } \\
\cline { 1 - 4 } Dilatada & $66,7 \%$ & $29,4 \%$ & $25 \%$ & \\
\hline
\end{tabular}

Con técnicas de anastomosis directa la tasa de estenosis oscila entre $0 \%{ }^{26}$ y $6,7 \%{ }^{9}$. Nuestros resultados son ligeramente superiores, $9 \%$, pero consideramos que la diferencia no es significativa

Contrariamente a los resultados comunicados por la literatura, el lado izquierdo, que precisa de mayor disección para trasponer o superponer sobre el colon descendente, no presenta mayor tasa de estenosis en nuestra experiencia.

\section{CONCLUSIONES}

- En nuestra experiencia la anastomosis menos estenosante es la tipo bivalva. 
- A mayor sencillez de técnica mejores resultados.

- La tasa de estenosis es siempre mayor cuando la vía esta dilatada previa a la derivación, independientemente de la técnica utilizada.

\section{REFERENCIAS}

1. O`REILLY PH.: Diuresis renography 8 years later: an update. J Urol 1986; 136: 993-995.

2. FITCHNER J.: Follow-up after urinary diversion. Urol Int 1999; 63: 40-45.

3. KRISTJANSSON A, WALLIN L, MANSSON W.: Renal function up to 16 years after conduit (efluxing or anti-reflux anastomosis) or continent urinary diversion. 1. Glomerular filtration rate and patency of uretero-intestinal anastomosis. $\mathrm{Br} J$ Urol 1995; 76: $539-545$.

4. FISCH M, WAMMACK R, STEINBACH F, MÜLLER SC, HOHENFELLNER R.: Sigma-Recto Pouch (Mainz Pouch II) Urol Clin North Am 1993; 20: 561-569.

5. PAGANO F, ARTIBANI W, ARAGONA F, BASSI P, RUFFATO A, MULONIA A.: La Vejiga Ileal Padovana (VIP): técnica quirúrgica, evaluación funcional a largo plazo, complicaciones y conducta a seguir. Arch Esp Urol 1997; 50: 785-793.

6. SANTUCCI RA, PARK CH, MAYO ME, LANGE PH.: Continence and urodynamic parameters of continent urinary reservoirs: comparision of gastric, ileal, ileocolic, rigth colon, and sigmoid segments. Urology 1999; 54: 252-257.

7. McGUIRE EJ, WOODSIDE JR, BORDEN TA et al.: Prognostic value of urodynamic testing in myelodysplasic patients. J Urol 1981; 126: 205-209.

8. PANTUCK AJ, HAN K-H, PERROTTI M, WEISS RE CUMMINGS KB.: Uretroeneteric anastomosis in continet urinary diversion: long-term results and complications of direct versus norefluxing techniques. J Urol 2000; 163: 450-455.

9. DE CARLI P, MICALI S, O'SULLIVAN D, MANIERO G, CUSUMANO G, FATTAHI H, CANCRINI A.: Ureteral anastomosis in the orthotopic ileal neobladder: comparision of 2 techniques. J Urol 1997; 157: 469-471.

10. ROTH S, VAN AHLEN H, SEMJONOW A, OBERPENNING F, HERTLE L.: Does the success of ureterointestinal implantation in orthotopic bladder substitution depend more on surgeon level of experience or choice of technique?. J Urol 1997; 157: 56-60.

11. HELAL M, POW-SANG J, SANFORD E, FIGUEROA E, LOCKHART J.: Direct (nontunneled) ureterocolonic reimplantation in association with continent reservoirs. J Urol 1993 sep;150 (3): 835-837.

12. LE DUC A, CAMEY M, TEILLAC P.: An original antireflux ureteroileal implantation technique: longterm follow-up. J Urol 1987; 137: 1156-1158.

13. KRISTJANSSON A, BAJC M, WALLIN L, WILLNER J, MANSSON W.: Renal function up to 16 years after conduit (efluxing or anti-reflux anastomosis) or continent urinary diversion. 2.- Renal scarring and location of bacteriuria. Br J Urol 1995; 76: 546550 .
14. HAUTMANN RE, DE PETRICONI R, GOTTFRIED HW, KLEINSCHMIDT K, MATTES R, PAISS T.: The ileal neobladder: complcations and funcional results in 363 patients after 11 years of follow-up. J Urol 1999; 161: 422-428.

15. THONEY HC, SONNENSCHEIN MJ, MADERSBACHER S, VOCK P, STUDER UE.: Is ileal orthotpic neobladder substitution with an afferet tubular segment detrimental to the upper urinary tract in the long term. J Urol 2002; 168: 2030-2034.

16. GOLBANO JM, SERRANO A, OTERO I, MERINO C, CHICHARRO J, GONZÁLEZ-PERAMATO P.: Ureterosigmoidostomía destubulizada Mainz II: ?una derivación con futuro?. Arch Esp de Urol 2001; 54: 123-128.

17. SKINNER SG, LIESKOVSKY G, BOYD S.: Continent urinary diversion. $J$ Urol 1989; 141: 1323-1327.

18. STUDER UE, SPIEGEL T, CASANOVA GA, SPRINGER J, GERBER E, ACKERMANN DK, GURTNER F, ZINGG EJ.: Ileal bladder substitue: antirreflux nipple or afferent tubular segment? Eur Urol 1991; 20: 315-326.

19. SHAABAN AA, GABALLAH MA, EL DIASTY TA, GHONEIM MA.: Urethral controled bladder substitution: a comparision between the intususcepted nipple valve and the techique of Le Duc as antirreflux porcedures. $J$ Urol 1992; 148: 1156-1161.

20. SCHWAIBOLD H, FRIEDRICH MG, FERNÁNDEZ S, CONRAD S, HULAND H.: Improvement of ureteroileal anastomosis in continent urinary diversion with modified Le Duc procedure. J Urol 1998; 160: 718-720.

21. MURAISHI O, YAMSHITA T, ISHIKAWA S, HARA Y, TOKUE A.: Improvement of ureteroileal anastomosis in oOrthotopic ileal neobladder with modified Le Duc procedure: short submucosal tunnel technique. J Urol 2001; 165: 798-801.

22. WENDEROTH UK, BACHOR R, EGGHART G et al.: The ieal neobladder: experience and resultas of more than 100 consecutive cases. J Urol 1990; 143: $492-495$.

23. STONE AR, McDERMOTT JP.: The split-cuff ureteral nipple reimplantation techique: reliable reflux prevention from bowel segments. $J$ Urol 1989; 142: 707-709.

24. SAGALOWSKY AI.: Further experience with splitcuff nipple ureteral reimplantation in urinary diversion. J Urol 1998; 159: 1843-1844.

25. MARINO G, CEVOLI R, LAUDI M.: Uretero-intestinal anastomosis using the ureteral nipple technique with personal modification in continent urinary diversión. Minerva Urol Nefrol 2000; 52: 183-187.

26. SOULIE M, DE PETRICONI R, GSHWEND J, HAUTMANN R, GNANN R.: The new uretero-ileal anastomosis technique in Hautmann ileal neobladder. Prog Urol 2001; 11: 29-33.

Dr. J.L. Moyano Calvo

C/ Bogotá 19, 3, 2ํㅡ

41013 Sevilla

(Trabajo recibido el 19 mayo 2003) 